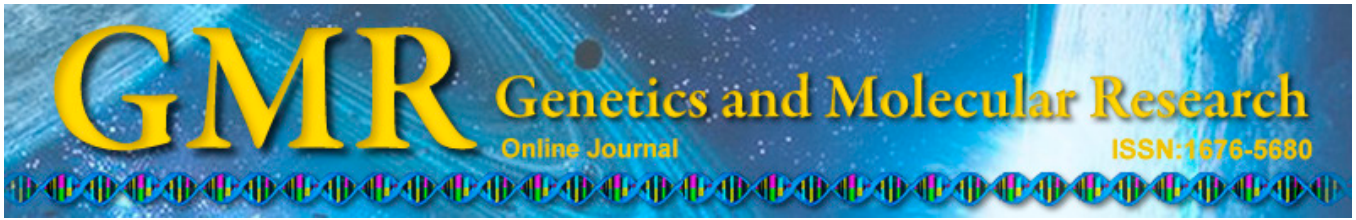

\title{
Identification of the porcine sialoadhesin gene promoter region and its cell-specific expression in porcine alveolar macrophage cells
}

\author{
W. Sun*, Z. Xie*, S. Zhang, W. Nan and J. Chen \\ College of Animal Science and Technology, Nanjing Agricultural University, \\ Nanjing, Jiangsu, China \\ *These authors contributed equally to this study. \\ Corresponding author: J. Chen \\ E-mail: jiechen@njau.edu.cn
}

Genet. Mol. Res. 13 (4): 9615-9621 (2014)

Received November 5, 2013

Accepted May 5, 2014

Published November 14, 2014

DOI http://dx.doi.org/10.4238/2014.November.14.5

\begin{abstract}
Porcine reproductive and respiratory syndrome (PRRS), which is caused by the PRRS virus (PRRSV), is a communicable disease. PRRS caused huge economic losses to swine breeding. The porcine alveolar macrophage (PAM) cell is the main target cell of PRRSV; therefore, it is very important to identify the specific gene promoter that controls expression in PAM cells so that the anti-PRRSV exogenous gene can be efficiently and specifically expressed in PAM cells to improve porcine resistance to PRRSV. In this study, the transcription initiation site for sialoadhesin (Siglec-1), which is a porcine alveolar macrophage-specific gene, was determined by 5 ' rapid amplification of cDNA end, and $88 \mathrm{bp}$ of the 5'-untranslated region was cloned. Siglec-1 promoter activity was detected by a dual-luciferase reporter assay, which showed that the fragment from -173 to $+81 \mathrm{bp}$ had the strongest promoter activity. Additionally, the cell-specific expression of the promoter fragments was tested in a PAM cell line (CRL-2844 cells), porcine
\end{abstract}


kidney 15 cell line (PK-15 cells), porcine fetal fibroblast (PEF) cells, and porcine preadipocytes. These results also showed that the fragment from -173 to $+81 \mathrm{bp}$ had the strongest cell-specific expression in PAM cells.

Key words: Porcine reproductive and respiratory syndrome; Porcine reproductive and respiratory syndrome virus; Promoter activity; Cell-specific expression; Siglec-1

\section{INTRODUCTION}

Porcine reproductive and respiratory syndrome (PRRS) is a viral infectious disease, which is accompanied by the clinical manifestations of respiratory problems in pigs of all ages and severe reproductive failure in sows that is characterized as the leading cause of late-term abortions and an increasing number of stillborn and weak pigs (Vanderheijden et al., 2003). This disease first appeared in the United States in 1987, and it broke out in Europe in 1989; then, it gradually spread to other regions around the world (Wensvoort et al., 1991; Collins et al., 1992). PRRS has caused huge economic losses for the world pig industry, it has become one of the major epidemics of large-scale pig farms, and it is also a major problem for disease control in the global swine industry (Chen et al., 2006).

PRRS virus (PRRSV) is a single-stranded RNA virus that belongs to the Arteriviridae family (Dea et al., 2000). In vivo, PRRSV infects the monocyte/macrophage lineage, primarily alveolar macrophages. It also infects macrophages in tonsils, spleen, liver, thymus, lymph nodes, and Peyer's patches (Duan et al., 1997a,b). Additional research showed that the virus also infects porcine testicular germ cells, whereas freshly isolated blood monocytes are hardly infected (Sur et al., 1996,1997). Porcine alveolar macrophages (PAMs) are the main target cells of PRRSV in the acute phase of infection (Teifke et al., 2001). The restricted cell tropism of PRRSV is determined by cellular membrane factors of the target cells (Kreutz, 1998).

PAMs are the target cells that are specifically infected by PRRSV in porcine. Thus, it is important to identify the promoters that are involved in PAM-specific gene expression, which could initiate the efficient and specific expression of anti-PRRSV exogenous genes in PAM cells. The Siglec-1 gene, which belongs to the lectin family (Varki and Angata, 2006), encodes an essential PRRSV receptor that mediates attachment and internalization on macrophages; it is highly expressed in PAM cells. In humans and mice, Siglec-1 is only expressed on discrete subsets of tissue macrophages, including the spleen, bone marrow, lymph nodes, colon, liver, and lung (Crocker and Gordon, 1989; Hartnell et al., 2001). In porcine, Siglec-1 is critical for the entry of PRRSV into PAMs, and it mediates the internalization of PRRSV into porcine kidney 15 (PK-15) cells, which are resistant to PRRSV entry and contain an exogenous Siglec-1 gene (Vanderheijden et al., 2003).

In this study, Siglec-1 promoters of different lengths were cloned, and their transcriptional activity was analyzed in a PAM cell line, CRL-2844. The cell-specific expression of the Siglec-1 promoter was verified in porcine preadipocyte, PK-15, porcine fetal fibroblast (PEF) and CRL-2844 cells by a dual-luciferase reporter assay system. 


\section{MATERIAL AND METHODS}

\section{Cell culture}

CRL-2844, PK-15, PEF, and porcine preadipocyte cells were used in this experiment. CRL-2844 cells were cultured in 10\% fetal bovine serum (FBS) and Roswell Park Memorial Institute 1640 (RPMI 1640) medium. PK-15 and PEF cells were cultured in 10\% FBS and Dulbecco's modified Eagle medium nutrient mixture F-12 (DMEM/F12). Porcine preadipocyte cells were cultured in 10\% FBS and DMEM. The culture medium was changed every 3 days.

\section{Determination of the transcription initiation site of Siglec-1}

The transcription initiation site of Siglec-1 was determined by 5 ' rapid amplification of cDNA end (5'-RACE). RNA was extracted from CRL-2844 cells using Trizol (Takara, Dalian, China). 5'-RACE was performed according to the SMART ${ }^{\text {TM }}$ RACE cDNA amplification kit instructions (Takara, Dalian, China). The 5' sequence of Siglec-1 was determined using the universal primer A mix (UPM) supplied by the kit (5'-CTAATACGACTCACTATAGGGCAA GCAGTGGTATCAACGCAGT-3') and a gene-specific primer (GSP) (5'-CACGTTTCTGG TCTTTGAGCTTCGTCC-3'). The 5'-RACE product was characterized by nested polymerase chain reaction (PCR) using the nested gene-specific primer (NGSP) (5'-GCTGATCTCA AAGCGGAAGTTATAGGA-3') and UPM. RACE products and nested PCR products were cloned into pMD-19T. The sequences were verified by sequencing.

\section{Analysis of promoter activity}

DNA was extracted from CRL-2844 cells. In order to test the promoter activity, 8 fragments (Table 1) of various lengths were amplified from the 5'-flanking region of the Siglec-1 gene with the introduction of XhoI and HindIII sites. The amplified products were digested and cloned into the multiple cloning site of the pGL3-basic vector, which contains the firefly luciferase gene as a reporter. The vectors were verified by restriction enzyme digestion and sequencing.

Eight constructed vectors and the invariant control vector pRL-TK, which contains the Renilla luciferase gene as a reporter, were used to co-transfect CRL-2844 cells using Lipofectamine ${ }^{\mathrm{TM}} 2000$. The pGL3-basic and pRL-TK vectors were co-transfected as a control. The culture medium was replaced with fresh RPMI 1640 medium after $6 \mathrm{~h}$, and the cell extracts were prepared $24 \mathrm{~h}$ after transfection by passive lysis buffer (PLB). Then, the promoter activity was determined using a dual-luciferase reporter assay kit (Promega, Madison, WI, USA) according to manufacturer instructions. Briefly, $20 \mathrm{~mL}$ PLB cell lysate was transferred to a tube containing $100 \mathrm{~mL}$ luciferase assay buffer II (LAB II) and mixed for 2-3 min. Then, the tube was placed in the luminometer, and the firefly luciferase activity measurement M1 was recorded. Next, $100 \mathrm{~mL}$ Stop \& Glo ${ }^{\circledR}$ substrate was added to the same tube and vortexed briefly. The tube was placed in the luminometer again, and the Renilla luciferase activity value M2 was recorded. The promoter activity was denoted by the value M1/M2. 
Table 1. Primer sequences for Siglec-1 vector construction.

\begin{tabular}{ll}
\hline Vector & Primer sequence (5'-3') \\
\hline pLUC173 & GCGCTCGAGAGAGGACTTTCTGGGGCATG \\
pLUC574 & GCGCTCGAGAGGACTGGCCCCAGAGTGA \\
pLUC691 & GCGCTCGAGGACCCCAAAAGGGACGTT \\
pLUC740 & GCGCTCGAGTGCTCACCAGGGACAAGG \\
pLUC901 & GCGCTCGAGTTGGGCAGGGTCCTTGTG \\
pLUC1444 & GCGCTCGAGTTTCTAGGGCCACTCCTGTG \\
pLUC2188 & GCGCTCGAGTCGTCTGTGGATGGACACTCGGAAAC \\
Common downstream primer & GCGCTCGAGTGCAGCTGCGGCTCAGATTCA \\
\hline
\end{tabular}

\section{Cell-specific analysis of the Siglec-1 promoter}

To test cell-specific expression of the Siglec-1 promoter, the promoter and pRL-TK vectors were co-transfected into CRL-2844, PK-15, PEF, and porcine preadipocyte cells. The relative promoter activity was measured by a dual-luciferase reporter assay kit.

\section{Statistical analysis}

All data are reported as means \pm SE. All experiments were performed 6 times. Tissuespecific activity of the promoter was analyzed with the independent $t$-test procedure (SPSS Inc., Chicago, IL, USA). The transcriptional activities of deletion promoters were compared with one-way analysis of variance (SPSS Inc.). P $<0.05$ was considered to be statistically significant.

\section{RESULTS}

\section{5'-untranslated region (UTR) of the porcine Siglec-1 gene}

The gene-specific PCR and nested PCR products of 5'-RACE were about $1000 \mathrm{bp}$ (Figure 1A) and $500 \mathrm{bp}$ (Figure 1B), respectively. The product of the 5'-RACE was sequenced and the result is shown in Figure 1C. Sequence alignment revealed $88 \mathrm{bp}$ before the start codon (Figure 1D).

\section{Promoter activity and cell-specific analysis}

The upstream $3500 \mathrm{bp}$ before the start codon was used in the promoter activity analysis. Eight fragments, which contained the sequences from -173 to $+81,-574$ to $+81,-691$ to $+81,-740$ to $+81,-901$ to $+81,-1444$ to $+81,-2188$ to +81 , and -3412 to $+81 \mathrm{bp}$, were cloned into pGL3-basic vectors, and the vectors were named pLUC173, pLUC574, pLUC691, pLUC740, pLUC901, pLUC1444, pLUC2188, and pLUC3412, respectively. The vectors were verified by restriction enzyme digestion. All products were consistent with the expected lengths of $254,655,772,950,982,1525,2268$, and $3493 \mathrm{bp}$.

The transcription activities of the deletion promoters are shown in Figure 2. All vectors except pLUC3412 showed significantly higher activity than the pGL3-basic vector. The pLUC173, pLUC574, and pLUC691 vectors had the strongest transcriptional activity of all vectors. However, the pLUC173 vector had the shortest promoter fragment. 
Because the pLUC173 vector had the highest activity for gene expression, we tested this promoter fragment for cell-specific expression. The results are shown in Figure 3. Among CRL-2844, PK-15, PEF, and porcine preadipocyte cells, the pLUC173 vector had the highest promoter activity in CRL-2844 cells.

A

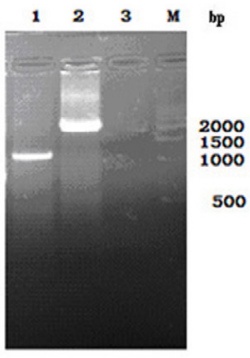

B

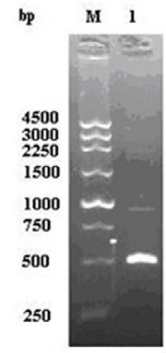

$\mathrm{C}$

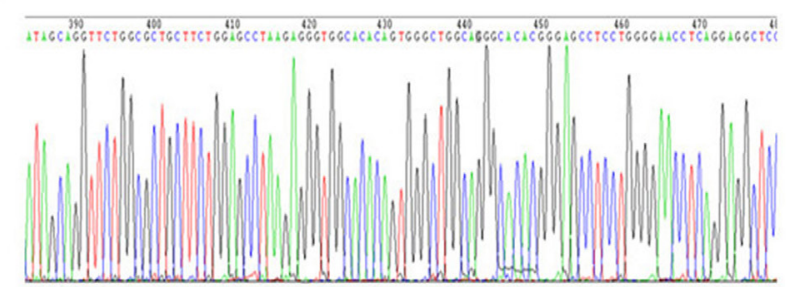

D

DNA sequence TGAGACCCAGG TACCCTGAGG TTCCCCAGGAGGCTCCCG TG TGCCCTGCCAGCCCACTGT RACE | | ||||||||||||||||||||||||||||||||||||||||||||||||||

GTGCCACCCTCTTAGGCTCCAGAAGCAGCGCCAGAACCTGCT || ||||||||||||||||||||||||||||||||||||||||||

Figure 1. Results of 5'-RACE. A. Agarose gel (1\%) image of 5'-RACE products. B. Agarose gel (1\%) image of nest PCR products. C. Map analysis of 5'-RACE sequencing production. D. Sequencing production of 5'-RACE.

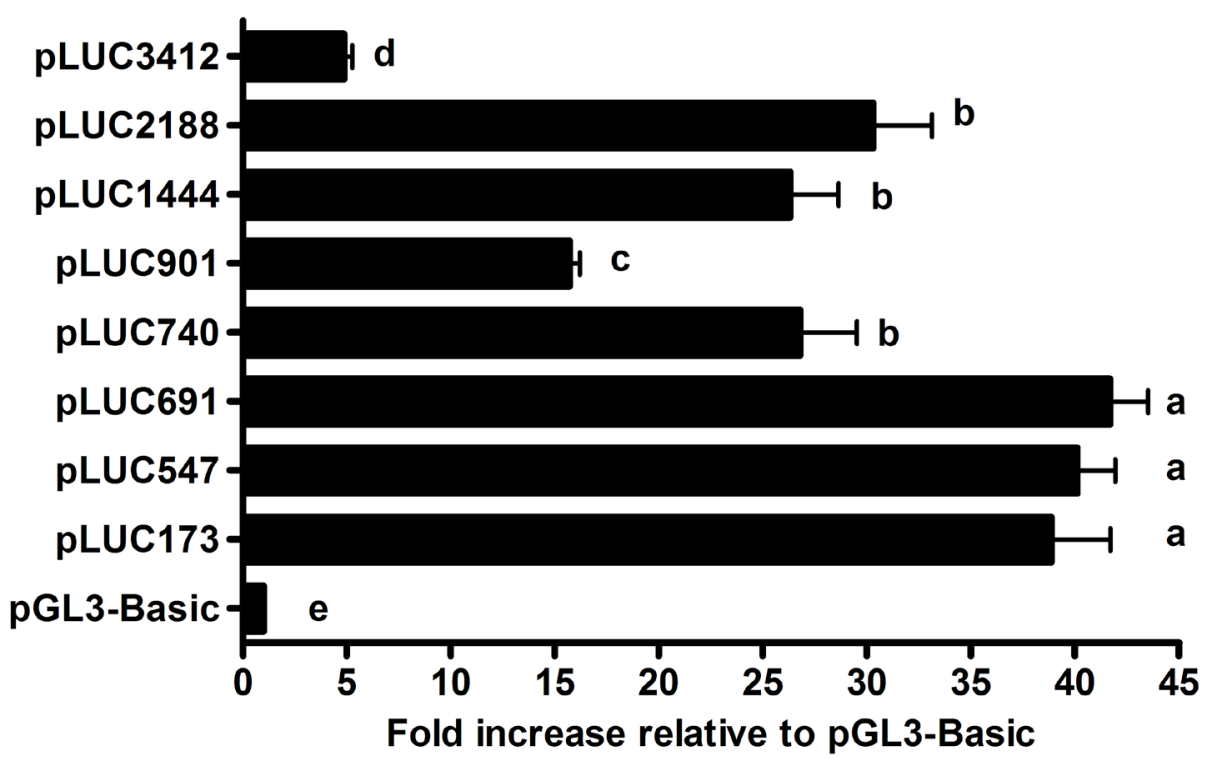

Figure 2. Promoter activity analysis. Means with different letters denotes significant difference $(\mathrm{P}<0.05)$. 


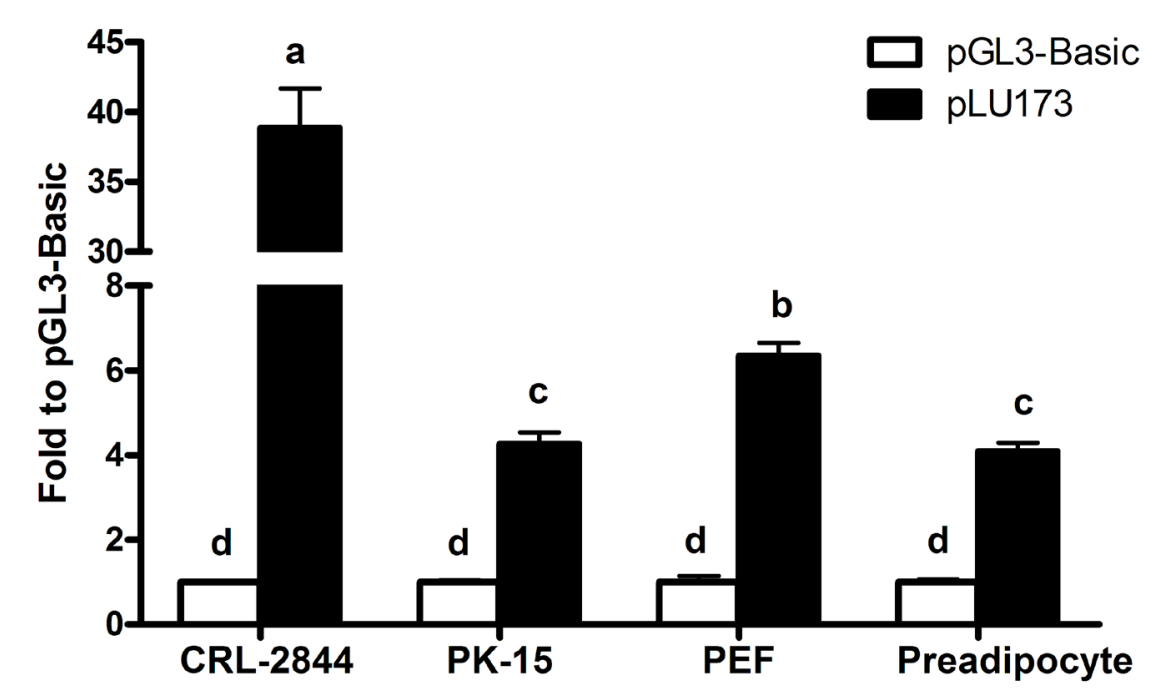

Figure 3. Cell-specific analysis of promoter fragments. Means with different letters denotes significant difference $(\mathrm{P}<0.05)$.

\section{DISCUSSION}

The complexity of the mammalian genome is the major obstacle to identifying cisregulatory elements. The development of transcription start site identification technology provides a reliable endorsement for gene promoter positions. 5'-RACE can rapidly amplify the $5^{\prime}$ end of mRNA using cDNA as a template. The products of this method have limited errors. Moreover, this method is highly efficient, fast, and sensitive. In this study, we obtained the Siglec-1 5'-UTR using 5'-RACE. The GSP and NGSP mapped to +865 and +360 bp after the start codon; therefore, the PCR products should be greater than 865 and $360 \mathrm{bp}$, respectively. The lengths of the 5'-RACE products, which were approximately 1000 and $500 \mathrm{bp}$, were consistent with the expected results. Thus, we successfully obtained the 5'-UTR of Siglec-1.

If a positive regulatory factor binds the promoter cis-regulatory element, the activity of the promoter is enhanced. In the opposite situation, if the promoter cis-regulatory element is bound by negative regulatory factors, the promoter activity is reduced. From the restriction enzyme digestion and sequencing, we successfully constructed deletion promoter expression vectors. The pLU740, pLU901, and pLU3412 vectors had lower promoter activity than the pLU691 and pLU2188 vectors. This result may indicate that there were negative regulatory factors in the regions from -691 to -901 and -2188 to $-3412 \mathrm{bp}$. The pLU173 promoter activity was 40 -fold higher than that of the control group, and this vector had the shortest promoter fragment. Hence, the core promoter was located in the region from -173 to -1 bp. The pLU173 vector had the highest promoter activity in PAM cells; hence, Siglec-1 had a cell-specific expression pattern in PAM cells. We hypothesize that PAM cell-specific transcription elements exist in the promoter region from -173 to -1 bp of Siglec-1. Additional research needs to be done to identify the transcription elements. In this study, we successfully identified the Siglec-1 cell-specific promoter region. This is helpful for improving porcine disease resistance and breeding. 


\section{ACKNOWLEDGMENTS}

Research supported by the National Key Genomic Transformation Program of China (\#2009ZX08009-143B). The authors also thank Professor Ping Jiang for help and support.

\section{REFERENCES}

Chen J, Liu T, Zhu CG, Jin YF, et al. (2006). Genetic variation of Chinese PRRSV strains based on ORF5 sequence. Biochem. Genet. 44: 425-435.

Collins JE, Benfield DA, Christianson WT, Harris L, et al. (1992). Isolation of swine infertility and respiratory syndrome virus (isolate ATCC VR-2332) in North America and experimental reproduction of the disease in gnotobiotic pigs. J. Vet. Diagn. Invest. 4: 117-126.

Crocker PR and Gordon S (1989). Mouse macrophage hemagglutinin (sheep erythrocyte receptor) with specificity for sialylated glycoconjugates characterized by a monoclonal antibody. J. Exp. Med. 169: 1333-1346.

Dea S, Gagnon CA, Mardassi H, Pirzadeh B, et al. (2000). Current knowledge on the structural proteins of porcine reproductive and respiratory syndrome (PRRS) virus: comparison of the North American and European isolates. Arch. Virol. 145: 659-688.

Duan X, Nauwynck HJ and Pensaert MB (1997a). Effects of origin and state of differentiation and activation of monocytes/ macrophages on their susceptibility to porcine reproductive and respiratory syndrome virus (PRRSV). Arch. Virol. 142: 2483-2497.

Duan X, Nauwynck HJ and Pensaert MB (1997b). Virus quantification and identification of cellular targets in the lungs and lymphoid tissues of pigs at different time intervals after inoculation with porcine reproductive and respiratory syndrome virus (PRRSV). Vet. Microbiol. 56: 9-19.

Hartnell A, Steel J, Turley H, Jones M, et al. (2001). Characterization of human sialoadhesin, a sialic acid binding receptor expressed by resident and inflammatory macrophage populations. Blood 97: 288-296.

Kreutz LC (1998). Cellular membrane factors are the major determinants of porcine reproductive and respiratory syndrome virus tropism. Virus Res. 53: 121-128.

Sur JH, Cooper VL, Galeota JA, Hesse RA, et al. (1996). In vivo detection of porcine reproductive and respiratory syndrome virus RNA by in situ hybridization at different times postinfection. J. Clin. Microbiol. 34: 2280-2286.

Sur JH, Doster AR, Christian JS, Galeota JA, et al. (1997). Porcine reproductive and respiratory syndrome virus replicates in testicular germ cells, alters spermatogenesis, and induces germ cell death by apoptosis. J. Virol. 71: 9170-9179.

Teifke JP, Dauber M, Fichtner D, Lenk M, et al. (2001). Detection of European porcine reproductive and respiratory syndrome virus in porcine alveolar macrophages by two-colour immunofluorescence and in-situ hybridizationimmunohistochemistry double labelling. J. Comp. Pathol. 124: 238-245.

Vanderheijden N, Delputte PL, Favoreel HW, Vandekerckhove J, et al. (2003). Involvement of sialoadhesin in entry of porcine reproductive and respiratory syndrome virus into porcine alveolar macrophages. $J$. Virol. 77: 8207-8215.

Varki A and Angata T (2006). Siglecs--the major subfamily of I-type lectins. Glycobiology 16: 1R-27R.

Wensvoort G, Terpstra C, Pol JM, ter Laak EA, et al. (1991). Mystery swine disease in The Netherlands: the isolation of Lelystad virus. Vet. Q. 13: 121-130. 